Paediatr. Paedolog. $2021 \cdot 56$ (Suppl 1):S33-S37 https://doi.org/10.1007/s00608-021-00888-4 Angenommen: 12. März 2021

๑ Der/die Autor(en) 2021

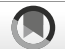

Elke Fröhlich-Reiterer ${ }^{1}$ S Sabine Hofer ${ }^{2} \cdot$ Hildegard Jasser-Nitsche $^{1} \cdot$ Ursula Lück $^{3}$ • Birgit Rami-Merhar ${ }^{4}$

'Universitätsklinik für Kinder- und Jugendheilkunde, Department für Allgemeine Pädiatrie, Medizinische Universität, Graz, Österreich

${ }^{2}$ Department für Pädiatrie 1, Medizinische Universität, Innsbruck, Österreich

${ }^{3}$ Abteilung für Kinder- und Jugendheilkunde, Thermenklinikum, Mödling, Österreich

${ }^{4}$ Universitätsklinik für Kinder- und Jugendheilkunde, Medizinische Universität, Wien, Österreich

\title{
Assoziierte Erkrankungen und andere Komplikationen
}

Die Inzidenz der Autoimmunthy-

\section{Assoziierte Autoimmun- erkrankungen}

Diabetesassoziierte Autoantikörper umfassen Inselzellantikörper (ICA), Insulin-Autoantikörper (IAA), Glutamatdecarboxylase-Antikörper (GAD), Tyrosinphosphatase-Antikörper (IA2) und Zinktransporter-8-Autoantikörper (ZnT-8), und ein oder mehrere Antikörper sind bei der Mehrzahl der Kinder mit Typ-1-Diabetes (T1D) nachzuweisen [1].

Kinder mit T1D haben ein höheres Risiko, auch andere organspezifische Autoantikörper (AK) (Schilddrüsen-AK, Zöliakie-AK, Parietalzell-AK oder Nebennieren-AK) und häufiger Autoimmunerkrankungen als die Normalbevölkerung, zu entwickeln [2]. Ein gemeinsamer genetischer Hintergrund, gepaart mit bestimmten Trigger-Faktoren, dürfte dafür verantwortlich sein.

Die beiden häufigsten assoziierten Autoimmunerkrankungen bei Kindern und Jugendlichen mit T1D sind die Autoimmunthyreoiditis und die Zöliakie.

Seltener können auch Antikörper gegen die Nebenniere und die Parietalzellen des Magens nachgewiesen werden.

\section{Autoimmunthyreoiditis - Hypothyreose}

Assoziierte Schilddrüsenerkrankungen sind die häufigste assoziierte Autoimmunerkrankung bei Kindern und Jugendlichen mit T1D [2, 3]. reoiditis liegt in der Normalbevölkerung zwischen 0,3-1,1 pro 100 Patientenjahren und bei Kindern und Jugendlichen mit T1D bei 3-8\%. Die Prävalenz der Autoimmunthyreoiditis nimmt mit dem Alter auf bis $\mathrm{zu} 20 \% \mathrm{zu}$, wobei bei den meisten Patienten eine Hypothyreose vorliegt [2]. Bereits im ersten Jahr der Diabetesdiagnose sind bei bis zu $29 \%$ der Patienten Schilddrüsen-AK (AntiThyreoperoxidase[TPO], Anti-Thyreoglobulin[TG]) nachweisbar [2]; diese gehen einer latenten oder klinisch manifesten Hypothyreose oft voraus. Mädchen sind häufiger betroffen als Buben, besonders während der Pubertät. Zusätzlich besteht eine Assoziation mit dem Alter und der Diabetesdauer [2, 3].

Klinische Symptome können Struma, Gewichtszunahme, verzögertes Wachstum, verzögerte Pubertätsentwicklung, Müdigkeit, Bradykardie, Kälteintoleranz und Lethargie sein. Sehr häufig verläuft diese Erkrankung aber auch asymptomatisch und wird nur im Screening festgestellt.

\section{Diagnose}

Bei verminderter Konzentration an fT4 und erhöhter Konzentration an TSH liegt eine manifeste Hypothyreose vor. Wenn lediglich das TSH erhöht ist, das fT4 aber im Normalbereich, dann spricht man von einer latenten Hypothyreose. Eine Ultraschalluntersuchung der Schilddrüse zeigt oft einen typischen Befund mit Vergrößerung der Schilddrüse, inhomogenen $\mathrm{Pa}$ - renchymveränderungen und Hypervaskularisierungszeichen.

\section{Therapie}

Die Therapie ist die Substitution von L-Thyroxin (『 Abb. 1; [4]). Eine Laborkontrolle alle (3 bis) 6 Monate und auch eine regelmäßige sonographische Kontrolle der Schilddrüse (alle 1-2 Jahre, bei Knotenbildung häufiger) ist indiziert [2].

Eine unbehandelte Hypothyreose kann zu einer Verschlechterung des Lipidprofils im Sinn einer Erhöhung von Cholesterin-, Low-densitylipoprotein(LDL) und Triglyzeridkonzentrationen führen.

\section{Autoimmunthyreoiditis - Hyperthyreose}

Die Hyperthyreose kommt, bei Kindern und Jugendlichen mit T1D deutlich seltener vor als die Hypothyreose, jedoch häufiger als in der Normalbevölkerung. Die Prävalenz wird mit 0,5-6\% angegeben $[2,5]$. Die Ursache der Hyperthyreose ist entweder die hyperthyreote Phase der Autoimmunthyreoiditis Hashimoto oder ein Morbus Basedow.

\section{Klinische Symptome}

Klinische Symptome sind Gewichtsverlust bei normalem Appetit, Agitation, Tachykardie, Tremor, Hitzewallungen, Schilddrüsenvergrößerung und Exophthalmus. Auch unerklärbare Blutzuckerschwankungen können ein Hinweis für eine Hyperthyreose sein [2]. 


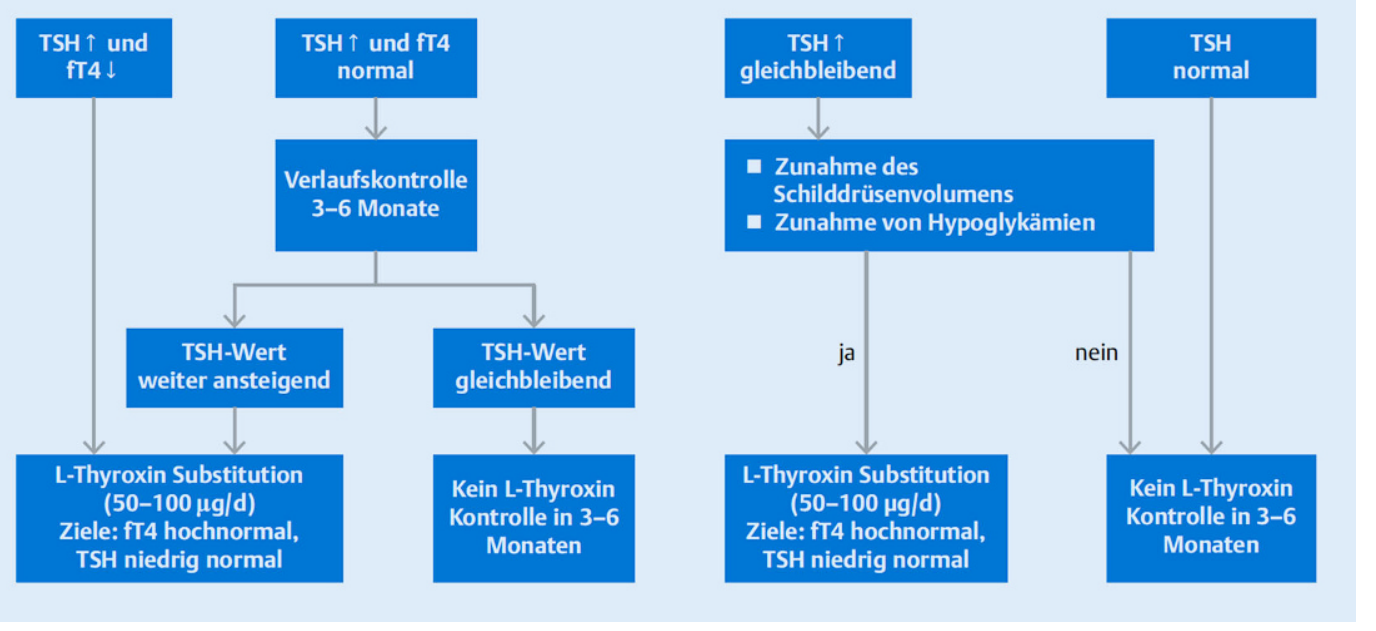

Abb. $1<$ Schema zur Behandlung der Hashimoto-Thyreoiditis. (Adaptiert nach [4])

\section{Diagnose}

Bei der Hyperthyreose ist die TSH-Konzentration supprimiert, die fT4-Konzentration erhöht, Schilddrüsen-AK (AntiTPO bei Hashimoto Thyreoiditis, Anti-TSH-Rezeptor bei Morbus Basedow) sind nachweisbar und eine Schilddrüsensonographie (Ausschluss eines Adenoms) sollte durchgeführt werden.

\section{Therapie}

Therapiert wird mit Thiamazol oder Carbimazol. In der akuten Phase können auch $\beta$-Blocker (z. B. Propranolol) zur Therapie der Tachykardie und der Agitiertheit notwendig sein.

\section{Zöliakie}

Die Prävalenz der Zöliakie bei Kindern mit T1D liegt zwischen 1-10\% mit einer Inzidenz von etwa 8 pro 1000 Patienten $[2,6,7]$ und ist damit höher als in der Normalbevölkerung. In einem internationalen Vergleich von 3 Kontinenten, in den 53.000 Kinder und Jugendliche mit T1D eingeschlossen wurden, zeigt sich eine durchschnittliche Prävalenz von 3,5\%, mit Prävalenzraten von $1,9 \%$ in den USA, $3,2 \%$ in Deutschland/Österreich bis zu $7,7 \%$ in Australien [2].

Das Risiko für eine Zöliakie ist invers mit dem Alter bei Erstmanifestation assoziiert, mit dem höchsten Risiko bei den Kindern, die vor dem 5. Lebensjahr an T1D erkranken [2, 7, 8]. Die meisten Fälle werden im ersten Jahr nach Diabeteserstmanifestation diagnostiziert, gefolgt von der 2-bis-5-Jahresperiode, wobei be- sonders Kleinkinder ein erhöhtes Risiko haben. Die Mehrheit der Fälle wird innerhalb der ersten 10 Jahre des Screenings diagnostiziert, aber es gibt vereinzelt auch Fälle, die erst im Erwachsenenalter diagnostiziert werden [2].

Bei Kindern mit T1D liegt meist eine asymptomatische Form der Zöliakie vor [2] und weist somit meist nicht die traditionellen Symptome wie Gedeihstörung, Durchfällen oder Bauchschmerzen auf. Wenn Kinder mit T1D Symptome wie Durchfälle, Bauchschmerzen, Blähungen, Aphten, Kleinwuchs, verzögerte Pubertätsentwicklung oder Anämie aufweisen, dann muss an eine Zöliakie gedacht werden. Bei der undiagnostizierten Zöliakie wurden vereinzelt vermehrte Hypoglykämien und ein sehr niedriger Insulinbedarf beschrieben [2]. Da die meisten Kinder mit T1D asymptomatisch sind, wird ein Screening auf CD empfohlen.

\section{Diagnose}

Zum Screening nach Zöliakie werden IgA-Antikörper (Tissue-Transglutaminase[tTG]-AK und/oder endomysiale AK [EMA]) empfohlen. Beide Tests haben eine Sensitivität und Spezifität von $>90 \%$ [2]. Ein IgA-Mangel muss ausgeschlossen werden, da sonst die Interpretation der Befunde verfälscht sein kann (falsch-negativ). Im Fall eines IgA-Mangels beurteilt man die tTG-IgG oder/und EMA-IgG [2].

$\mathrm{Da}$ die Mehrzahl der Patienten mit T1D HLA-DQ2- und/oder DQ8-positiv sind, wird durch die Humanes-
Leukozytenantigen(HLA)-Testung nur ein kleiner Teil der Risikopatienten ausgeschlossen und die HLA-Typisierung kann als Screening-Methode nicht empfohlen werden, da sie weder praktisch noch kosteneffektiv ist [2].

Bei positiven AK, soll diese Bestimmung wiederholt werden. Wenn die Antikörper zweimal positiv sind, sollte das Kind einem pädiatrischen Gastroenterologen zugewiesen werden.

Die aktuellen Guidelines der European Society for Paediatric Gastroenterology Hepatology and Nutrition (ESPGHAN) aus dem Jahr 2020 empfehlen, dass bei Patienten mit hochpositiven tTG-AK (über das 10-Fache des oberen Normbereichs) auf eine Dünndarmbiopsie verzichtet werden kann [10]. In diesen neuen Guidelines wird aber nicht speziell auf die Gruppe der Kinder und Jugendlichen mit T1D eingegangen.

Die Arbeitsgruppe Pädiatrische Endokrinologie und Diabetologie Österreich (APEDÖ) empfiehlt derzeit bei asymptomatischen Kindern mit T1D und positiven tTG-AK (unabhängig von der Höhe der AK) weiterhin die Duodenalbiopsie, um anhand der Marsh-Klassifikation die Diagnose zu bestätigen [9]. Bei Kindern mit Symptomen und hochpositiven tTG-AK (über das 10-Fache des oberen Normbereichs) und positiven EMA kann in Absprache mit den pädiatrischen Gastroenterologen und der Familie eventuell auf eine Biopsie verzichtet werden. 


\section{Therapie}

Die Therapie besteht in der glutenfreien Diät (GFD). Kinder mit der Diagnose Zöliakie und deren Familien sollen eine Schulung bezüglich GFD und Unterstützung durch einen erfahrenen Diätologen/ eine erfahrene Diätologin erhalten. Unter einer glutenfreien Diät normalisiert sich die Dünndarmmucosa und die AK werden negativ. Ein weiteres Antikörperscreening ist somit als Verlaufsparameter bezüglich Einhaltung der glutenfreien Diät sinnvoll. Auch bei asymptomatischen Kindern, mit durch eine Biopsie gesicherter Zöliakie, ist eine glutenfreie Ernährung gerechtfertigt, um das Risiko für gastrointestinale Tumoren, Osteoporose und Eisenmangelanämie zu reduzieren [2]. Weiterhin zeigten rezente Studien, dass eine langjährige Zöliakie mit einem erhöhten Risiko für Retinopathie assoziiert ist und dass Patienten mit schlechter Compliance der GFD ein erhöhtes Risiko für eine Mikroalbuminurie haben können [2, 11].

\section{Morbus Addison}

Bis $\mathrm{zu} 2 \%$ der Patienten mit T1D haben nachweisbare Antiadrenale AK [2]. Morbus Addison, die primäre Nebenniereninsuffizienz, kann auch bei T1D mit dem Autoimmun-PolyglanduläremSyndrom (APS I und II) assoziiert sein. Das APS I ist auch als Autoimmun-Polyendokrinopathie-Candidiasis-Ektodermale-Dystrophie-Syndrom (APECED) bekannt [2].

\section{Klinische Symptome}

Klinische Symptome des M. Addison sind häufige Hypoglykämien, unerklärliche Abnahme des Insulinbedarfs, vermehrte Hautpigmentierung, Mattigkeit, Gewichtsverlust, Hyponatriämie, Hyperkaliämie, niedriger Blutdruck.

\section{Diagnose}

Beweisend sind ein geringer Kortisolanstieg im Adrenocorticotropin(ACTH)Test, erhöhtes ACTH und der Nachweis von Nebennierenrinden-AK (21Hydroxylase-AK).

\section{Therapie}

Therapiert wird durch Substitution von Glukokortikoiden, eventuell mit zusätzlicher Substitution von Mineralkortikoiden [2].

\section{Autoimmungastritis}

Parietalzellantikörper (PCA) sind Marker einer Autoimmungastritis und gegen die $\mathrm{H}^{+} / \mathrm{K}^{+}$-ATPase der Magenbelegzellen gerichtet. Die chronische Zerstörung der Protonenpumpe führt zu Eisenmangelanämie durch verminderte Säureproduktion und verminderte Eisenresorption. PCA inhibieren auch die Sekretion des Intrinsic-Faktors, was zu einem Vitamin-B12-Mangel und zu einer perniziösen Anämie führen kann [12].

Die Prävalenz der PCA bei Kindern und Jugendlichen mit T1D liegt zwischen 5,3-7,5\%. Ärzte sollen bei Vorliegen eines unklaren Eisenmangels, einer perniziösen Anämie und/oder von gastrointestinalen Symptomen an das mögliche Vorliegen einer Autoimmungastritis denken. Ein generelles Screening wird nicht empfohlen [2].

\section{Typ-1-Diabetes-bedingte Hautveränderungen}

\section{Lipodystrophien (Lipohypertro- phie und -atrophie)}

Lipohypertrophien findet man sehr häufig, da gerade Kinder dazu neigen, immer die gleichen Stichstellen zu verwenden. Lipohypertrophien sind assoziiert mit einem höheren $\mathrm{HbA1c}$, vielen Injektionen und einer längeren Diabetesdauer, nicht jedoch mit der Nadellänge. Seltenes Wechseln der Injektionsstellen, mehrfaches Wiederverwenden der Nadel und zu wenig Rotation im Bereich der Injektionsstellen sind Risikofaktoren für die Entstehung einer Lipohypertrophie. Die Insulinresorption an diesen Stellen ist vermindert und meist verzögert [2]. Die Therapie der Lipohypertrohie ist das Meiden der betroffenen Stelle für 2 bis 3 Monate.

Die Lipoatrophie ist eine Form der lokalisierte Lipodystrophie und eine bekannte Komplikation der Insulintherapie und ist charakterisiert durch den Verlust des subkutanen Fetts. Seit der Einführung des Humaninsulins werden Lipoatrophien an den Stellen der Insulinapplikation seltener beobachtet (unter 1-2\% der Patienten mit T1D), haben aber mit den Insulinpumpentherapien wieder gering zugenommen [2]. Der Mechanismus, der zur Lipoatrophie führt, ist noch nicht vollständig bekannt, aber es scheint eine Immunpathogenese zu sein. Risikofaktoren für die Entstehung einer Lipoatrophie sind das Nichtrotieren der Katheteranlagestelle bei Insulinpumpentherapie oder das mehrfache Wiederverwenden der Nadel bei Pen-Therapie [2]. Die Therapien sind limitiert. Empfehlungen sind das Wechseln der Injektions- bzw Katheteranlagestellen und die Verwendung von Insulinanaloga. Unter der Therapie mit Steroiden (oral oder per injectionem) kam es in Fallberichten zu Rückgängen der Lipoatrophie [2].

\section{Hautveränderungen infolge von Continuous Glucose Monitoring und Insulinpumpentherapie}

Durch die Verwendung von Continuous Glucose Monitoring (CGM) und Insulinpumpen kann es zu einem zusätzlichen Risiko für Hautirritationen kommen. Das hat besonders bei Kleinkindern eine große Bedeutung, da sie eine kleinere Fläche zur Verfügung haben.

Schlechte Desinfektion beim Kathetersetzen und längere Liegedauer des Katheters bergen ein Risiko für Infektionen und Abszesse. Dem kann durch gute Desinfektion und regelmäßiges Wechseln des Katheters (mindestens alle 3 Tage) vorgebeugt werden.

Bei Verwendung von CGM kann es zu Hautirritationen (Ekzeme oder auch Kontaktallergien auf Sensor und/oder Pflaster und/oder Kleberstoffe) kommen. Patienten sollen deshalb instruiert werden, die Sensorstellen zu rotieren. Bei allergischen Reaktionen können transparente Pflaster oder Hautschutzfilmsprays zum Einsatz kommen. Ekzeme können laut Guidelines mit Hautpflege und topischen Steroiden therapiert werden [2]. 


\section{Vitiligo}

Diese erworbene Pigmentierungsstörung ist charakterisiert durch den Verlust von Melanozyten. Das resultierende klinische Bild sind depigmentierte weiße Flecken oder die Leukodermie. Die Vitiligo tritt bei rund 1-7\% der Kinder und Jugendlichen mit T1D auf im Vergleich von 0,2 bis $1 \%$ in der Normalbevölkerung. Bis dato gibt es keine effektive Therapie. Patienten sollen darauf hingewiesen werden, dass sie die Sonne meiden und Sonnencremes verwenden sollen. Der Vitamin-D-Spiegel soll gemessen werden und bei niedrigen Spiegeln soll eine Supplementation überlegt werden, da Patienten mit Vitiligo häufig einen Vitamin-D-Mangel aufweisen [2].

\section{Necrobiosis lipoidica diabeticorum}

Unabhängig von der Qualität der Stoffwechseleinstellung treten diese Hautveränderungen bei Jugendlichen mit einer Prävalenz von 0,06 bis $1,6 \%$ auf. Es handelt sich um eine atrophische Dermatitis, meist im Bereich der Schienbeine beidseits. Sie ist charakterisiert durch kleine rundliche, rötliche Papeln, die sich zu größeren scharf begrenzten Plaques entwickeln, zentral erscheinen sie gelblich, teilweise kommt es zu Ulzerationen. Die Ätiologie ist unklar, eine Mikroangiopathie könnte eine ursächliche Rolle spielen. Die Therapie ist herausfordernd und inkludiert initial topische Steroide, bei Nichtansprechen eventuell intraläsionale oder systemische Steroide, jedoch mich variablen Erfolgen. Manche Autoren berichten über positive Effekte bei Raucherentwöhnung und Verbesserung der Stoffwechselkontrolle [2].

\section{Wachstum und Entwicklung}

Die Dokumentation von Wachstum und Entwicklung mittels Perzentilenkurven und Tanner-Stadien ist ein essenzieller Teil der Langzeitbetreuung von Kindern und Jugendlichen mit T1D.

Zum Zeitpunkt der Manifestation wurde ein beschleunigtes Längenwachstum beobachtet [13, 14]; die Ursache dafür ist unklar. Bei Patienten mit schlechter metabolischer Einstellung wurde in einigen Studien ein Abfall des Längenwachstums beobachtet [14], während Patienten mit einer besseren metabolischen Einstellung ein normales Wachstum aufwiesen.

Eine schlechte körperliche Entwicklung (Länge bzw. Gewicht), eine Hepatomegalie (nichtalkoholische Steatosis hepatis) sowie eine verzögerte Pubertätsentwicklung (Mauriac-Syndrom) wurde bei Kindern mit chronisch schlechter metabolischer Einstellung beobachtet.

Kinder mit T1D haben erhöhte Human-Growth-Hormone(hGH)-Spiegel und erniedrigte Insulin-like-growthfactor(IGF)-1-Spiegel. Der vermehrte Einsatz von physiologischeren Therapien, wie Insulinpumpen- und BasisBolus-Therapie, hat physiologischere Insulinspiegel zur Folge. Dies hat zu einer Verbesserung der GH/IGF-1-Achse und zu besseren Endlängen, unabhängig von der glykämischen Kontrolle, geführt [2].

In der Pubertät und vor allem bei Mädchen, besonders bei jenen, die in der $\mathrm{Pu}$ bertät an T1D erkranken und mit intensivierten Therapien behandelt werden, wird häufig eine vermehrte Gewichtszunahme beobachtet $[2,15]$. Ursächlich könnte eine $\mathrm{zu}$ hohe Insulindosis sein, die in der Pubertät zwar meist notwendig ist, aber nach Beendigung der Pubertät wieder reduziert werden muss.

Mädchen mit T1D haben ein höheres Risiko für Übergewicht und für die Entwicklung von Essstörungen [2]. Kinder und Jugendliche mit T1D und Übergewicht haben eine erhöhte Prävalenz für kardiovaskuläre Risikofaktoren (Hypertonie, Dyslipidämie). Bei übergewichtigen Mädchen besteht auch ein erhöhtes Risiko für die Entwicklung eines polyzystischen Ovarsyndroms (PCOS). Rezente Studien zeigen, dass eine zusätzliche Therapie mit Metformin die Serumandrogenspiegel gegenüber einer Placebogruppe deutlich reduzieren [2].

\section{Knochen}

T1D ist mit Osteoporose und einem erhöhten Frakturrisiko assoziiert, obwohl es nur wenige Daten für junge Patienten mit T1D gibt. Ein abnormes Knochenwachstum (Dichte und Qualität) bei Patienten mit T1D dürfte multifaktoriell sein. Komorbiditäten, wie Zöliakie oder Hypothyreose können die Knochengesundheit noch zusätzlich negativ beeinflussen, wobei das Ausmaß im Kindesund Jugendalter noch unklar ist.

Deshalb sollten Osteodensitometrien bei Risikopatienten (zusätzliche Zöliakie, Hypothyoreose, dunkle Haut) mit langer Diabetesdauer im späten Jugendalter in Betracht gezogen werden. Ein Screening auf Vitamin-D-Insuffizienz sollte speziell in Risikogruppen durchgeführt werden und bei Bestätigung eines Vitamin-DMangels sollte laut Guidelines substituiert werden [2].

\section{Zahngesundheit}

Kinder und Jugendliche mit T1D haben, vor allem bei schlechter Stoffwechseleinstellung, ein erhöhtes Risiko für Zahnprobleme wie Gingivitis, Karies und orale Infektionen. Ursachen dafür sind ein reduzierter Speichelfluss durch hohe Blutzuckerwerte und der vermehrte Bedarf an Süßigkeiten zur Therapie von Hypoglykämien. Deshalb sollte der regelmäßige Zahnarztbesuch Teil der Präventionsmaßnahmen sein [2].

\section{Screening-Empfehlungen}

- Regelmäßige Messung von Länge und Gewicht (Perzentilen), Blutdruck und Beurteilung der Pubertätsentwicklung (Tanner-Stadien)

- Screening auf zusätzliche Schilddrüsenfunktionsstörungen:

- Bei T1D-Erstmanifestation: basales TSH, fT4, fT3 und TPO-AK

- Danach alle 2 Jahre bei asymptomatischen Patienten ohne Struma

- Bei Symptomen häufiger

- Bei vor allem Hyperthyreose Bestimmung von Thyreotropin-Rezeptor-Autoantikörpern (TRAK)

- Screening auf Zöliakie

- Bei T1D-Erstmanifestation: mittels IgA, tTG-IgA und EMA

- Ein Screening auf IgA-Mangel sollte bei Erstmanifestation des T1D durchgeführt werden. Bei bestätigtem IgA-Mangel sollte das Zöliakie-Screening mit IgG-spezifischen Tests (tTG-IgG und/oder EM-IgG) durchgeführt werden 
- Danach alle 1-2 Jahre (laut ISPAD nach 2 und 5 Jahren)

- Bei klinischen Symptomen oder erstgradigen Verwandten häufiger

- Bei 2-mal positiven AK: Transfer an einen pädiatrischen Gastroenterologen und Dünndarmbiopsie (Marsh-Klassifikation)

- Bei bioptisch gesicherter Zöliakie: glutenfreie Diät, Schulung durch einen erfahrenen Diätologen

- Regelmäßige Überprüfung der Haut, hier vor allem die Injektions-, Katheter- und Sensorstellen

- Screening auf Vitamin-D-Mangel sollte, speziell in Risikogruppen (zusätzliche Zöliakie, Hypothyreose, dunkle Haut), erwogen werden

- Bei klinischen Symptomen sollten Ärzte an das mögliche Vorliegen eines M. Addison oder einer Autoimmungastritis denken und eine weitere Abklärung durchführen

Die Grundlage dieser Leitlinie sind die Empfehlungen der ISPAD-Guidelines [2].

\section{Korrespondenzadresse}

\section{Elke Fröhlich-Reiterer}

Universitätsklinik für Kinder- und Jugendheilkunde, Department für Allgemeine Pädiatrie, Medizinische Universität

Graz, Österreich

elke.froehlich-reiterer@medunigraz.at

Funding. Open access funding provided by Medical University of Graz.

\section{Einhaltung ethischer Richtlinien}

Interessenkonflikt. E. Fröhlich-Reiterer, S. Hofer H. Jasser-Nitsche, U. Lück und B. Rami-Merhar geben an, dass kein Interessenkonflikt besteht.

Für diesen Beitrag wurden von den Autoren keine Studien an Menschen oder Tieren durchgeführt. Für die aufgeführten Studien gelten die jeweils dort angegebenen ethischen Richtlinien.

Open Access. Dieser Artikel wird unter der Creative Commons Namensnennung 4.0 International Lizenz veröffentlicht, welche die Nutzung, Vervielfältigung Bearbeitung, Verbreitung und Wiedergabe in jeglichem Medium und Format erlaubt, sofern Sie den/die ursprünglichen Autor(en) und die Quelle ordnungsgemäß nennen, einen Link zur Creative Commons Lizenz beifügen und angeben, ob Änderungen vorgenommen wurden.
Die in diesem Artikel enthaltenen Bilder und sonstiges Drittmaterial unterliegen ebenfalls der genannten Creative Commons Lizenz, sofern sich aus der Abbildungslegende nichts anderes ergibt. Sofern das betreffende Material nicht unter der genannten Creative Commons Lizenz steht und die betreffende Handlung nicht nach gesetzlichen Vorschriften erlaubt ist, ist für die oben aufgeführten Weiterverwendungen des Materials die Einwilligung des jeweiligen Rechteinhabers einzuholen.

Weitere Details zur Lizenz entnehmen Sie bitte der Lizenzinformation auf http://creativecommons.org/ licenses/by/4.0/deed.de.

\section{Literatur}

1. Eisenbarth GS, Gottlieb PA (2004) Autoimmune polyendocrine syndromes. N Engl J Med 350(20):2068-2079

2. Mahmud FH, Elbarbary NS, Fröhlich-Reiterer $E_{,}$ Holl RW, Kordonouri O, Knip M, Simmons K Craig M (2018) Other complications and diabetesassociated conditions in children and adolescents. Pediatr Diabetes 19(Suppl 27):275-286

3. Kordonouri $O$ et al (2005) Natural course of autoimmune thyroiditis in type 1 diabetes: association with gender, age, diabetes duration, and puberty. Arch Dis Child 90(4):411-414

4. Neu A, Bürger-Büsing J, Danne T, Dost $A$ et al (2019) Diagnostik, Therapie und Verlaufskontrolle des Diabetes mellitus im Kindes -und Jugendalter. Diabetol Stoffwechs 14(Suppl 2):153-155

5. Dost A, Rohrer TR, Fröhlich-Reiterer E, Bollow $E_{,}$ Karges B, Böckmann A, Hamann J, Holl RW, DPV Initiative and the German Competence Network Diabetes Mellitus (2015) Hyperthyroidism in 276 children and adolescents with type 1 diabetes from Germany and Austria. Horm Res Paediatr 84(3):190-198

6. Crone Jet al (2003) Prevalence of celiac disease and follow-up of EMA in children and adolescents with type 1 diabetes mellitus. J Pediatr Gastroenterol Nutr 37(1):67-71

7. Fröhlich-Reiterer EE, Kaspers $S$, Hofer $S$ et al (2011) Anthropometry, metabolic control and follow-up in children and adolescents with type 1 diabetes mellitus and biopsy proven celiac disease: longitudinal data from a German/Austrian multicenter survey. J Pediatr 158(4):589-593

8. Cerutti F et al (2004) Younger age at onset and sex predict celiac disease in children and adolescents with type 1 diabetes: an Italian multicenter study. Diabetes Care 27(6):1294-1298

9. Marsh MN, Crowe PT (1995) Morphology of the mucosal lesion in gluten sensitivity. Baillieres Clin Gastroenterol 9(2):273-293

10. Husby S, Koletzko S, Korponay-Szabó I, Kurppa K, Mearin ML, Ribes-KoninckxC, Shamir R, Troncone R, Auricchio R, Castillejo G, Christensen R, Dolinsek J, Gillett $P$, Hróbjartsson A, Koltai T, Maki $M$, Nielsen SM, Popp A, Størdal K, Werkstetter K, Wessels M (2020) European Society Paediatric Gastroenterology, Hepatology and Nutrition Guidelines for Diagnosing Coeliac Disease 2020 JPediatr Gastroenterol Nutr 70:141-157

11. RohrerTR, WolfJ, LiptayS, Zimmer KP, Fröhlich-Reiterer E, Scheuing N, Marg W, Stern M, Kapellen TM, Hauffa BP, Wölfle J, Holl RW, DPV Initiative and the German BMBF Competence Network Diabetes Mellitus (2015) Microvascular complications in childhood-onset type 1 diabetes and celiac disease: a multicenter longitudinal analysis of 56,514 patients from the German-Austrian DPV database. Diabetes Care 38(5):801-807

12. De Block CE et al (2001) Beta-cell, thyroid, gastric, adrenal and coeliac autoimmunity and HLADQ types in type 1 diabetes. Clin Exp Immunol 126(2):236-241

13. Donaghue KC et al (2003) Secular trends in growth in diabetes: are we winning? Arch Dis Child 88(2):151-154

14. Holl RW et al (1998) Age at onset and long-term metabolic control affect height in type-1 diabetes mellitus. Eur JPediatr 157(12):972-977

15. Fröhlich-Reiterer EE, Rosenbauer J, Bechtold-Dalla Pozza S, Hofer SE, Schober E, Holl RW, DPV-Wiss Study Group and German BMBF Competence Networks Diabetes mellitus and Obesity. (2014) Predictors of increasing BMI during the course of diabetes in children and adolescents with type 1 diabetes: data from the German/Austrian DPV multicentre survey. Arch Dis Child 99(8):738-743 (Aug)

Hinweis des Verlags. Der Verlag bleibt in Hinblick auf geografische Zuordnungen und Gebietsbezeichnungen in veröffentlichten Karten und Institutsadressen neutral. 